\title{
Spectrum Sensing for Cognitive Networks Based on Dimensionality Reduction and Random Forest
}

\author{
Xin Wang ${ }^{1,2}$, Jin-Kuan Wang ${ }^{1, *}$, Zhi-Gang Liu ${ }^{1}$, Bin Wang ${ }^{1}$ and $\mathrm{Xi} \mathrm{Hu}{ }^{1}$ \\ ${ }^{I}$ College of Information Science and Engineering, Northeastern University, Shenyang \\ 110819, China \\ ${ }^{2}$ School of Information \& Control Engineering, Shenyang Jianzhu University, \\ Liaoning, Shenyang, 110168, China; \\ wjk@neuq.edu.cn,wangxin7988@163.com,zliu@mail.neuq.edu.cn, \\ wangbinneuq@qq.com,neuqhx@126.com
}

\begin{abstract}
In this paper, we study spectrum sensing based on dimensionality reduction and random forest $(R F)$ in low signal-to-noise ratio environments. Classifications of three digital modulation types, including BPSK, OFDM and 2FSK, are investigated. From the received radio signal, a set of cyclic spectrum features are first calculated, and the principal component analysis (PCA) is applied to extract the most discriminant feature vector for classification. Furthermore, the detecting signal is classified by the trained random forest, which uses the Gini index as the classification criteria, to test whether the primary user exists. The performance of our proposed PCA combining with RF algorithm is evaluated through simulations and compared with MME, SVM, RF. Experimental results show that with dimensionality reduction, the performance of classification is much better with fewer features than that of without dimensionality reduction.
\end{abstract}

Keywords: Cognitive networks, spectrum sensing, principal component analysis, random forest

\section{Introduction}

Cognitive network $(\mathrm{CN})$ viewed as a novel form of wireless communications, has been proposed to become a tempting solution to the spectrum underutilization problem. $\mathrm{CN}$ is the wireless communication system with intelligence, which senses its outside electromagnetic environment and learns from the surroundings, then adapts its internal states by changing certain operating parameters such as transmit-power, carrier-frequency, and modulation strategy in order to adapt to the change of its environment $[1,2]$.

Spectrum sensing is a cornerstone in cognitive networks, which detects the availability of radio frequency bands for possible use by secondary user without interference to primary user. As one of the key technologies of cognitive network, spectrum sensing can detect situation of spectrum utilization quickly, accurately, and effectively. Some traditional techniques proposed for spectrum sensing are energy detection, matched filter detection, cyclostationary feature detection $[3,4]$. In view of the radio channel complex environment, spectrum sensing technology focused on spectrum detection problem under low signal-tonoise ratio circumstances of channel multi-path fading and shadow fading. In [5], the authors proposed spectrum sensing algorithm based on the maximum-minimum eigenvalue (MME), but spectrum sensing effect of the algorithm is not ideal; detection rate is low in the low SNR. The cyclic spectral coherence is widely used to classify modulated signals. However, the high 
dimensionality of the spectral coherence function (SCF) representation makes it difficult to be directly applied in the signal identification algorithms. In $[6,7]$, the authors proposed the idea of projecting the SCF of the signal to cyclic domain profile and picking the peak values to determine the modulation format. However, the method is not ideal when the SNR is low, because some important information is lost. There has been a growing interest in applying machine learning algorithms to $\mathrm{CN}$ in recent years [8]. In [9], the authors proposed an ANNbased cognitive engine that learns how environmental measurements and the status of the network affect its performance on different channels. HMM as a classifier has been used in spectrum sensing. In [10], the authors designed a channel status predictor using a MFNN model. The SVM, developed by Vapnik and others [11], has been used for many machine learning tasks such as pattern recognition and object classifications. A SVM-based spectrum sensing algorithms proposed in [12] was applied to the field of cognitive radio, but the algorithms affect the classification effect directly due to the high computational complexity, to large-scale training samples, prone to over fitting and other reasons.

Random forest (RF) was coined by Leo Breiman in 2001 on the foundation of machine learning theory [13]. Random forest is a combination of tree predictors such that each tree depends on the values of a random vector sampled independently and with the same distribution for all trees in the random forest. It denotes that the error of the random forest algorithm is more stable, and the algorithm can overcome the disadvantages of a single decision tree. Random forest algorithm reflects the advantages of the strong classifier synthesized by multiple weak classifiers.

PCA [14] is the best-known linear dimensionality reduction method. Applying the PCA method to these spectral coherence features can decrease the classification errors in low SNR scenarios and reduce computational complexity effectively. In view of their advantages, we introduce a new spectrum sensing scheme, which applies PCA on the cyclostationarity features and obtains the first one or more principal components to form the principle cyclic spectrum(PCS) feature vector, then generates random forest(RF) to classify the detecting signal.

The remainder of the paper is organized as follows. In Section 2, the system model is described. The extraction of characteristic parameters is introduced in Section 3. The procedure of combining PCA dimensionality reduction with RF is revisited in Section 4 and is well validated with computer simulation in Section 5. The several concluding remarks are made in Section 6.

\section{System Model}

For simplicity, we assume that a cognitive radio network with one primary user and $\mathrm{W}$ secondary users, for any one of the secondary user, the presence of the primary user can be summarized as a hypothesis test model of two elements

$$
\left\{\begin{array}{c}
H_{0}: y(t)=n(t) \\
H_{1}: y(t)=s(t)+n(t)
\end{array}\right.
$$

Where $\mathrm{H}_{0}$ denotes that the primary user is not exist, $H_{1}$ denotes that the primary user

exists, $0 \leq t \leq T, T$ represents the sampling time of the received signal. A process $s(t)$ (the primary user signal) is said to be cyclostationary in wide sense if its mean and variance are 
periodic with a period $T ;{ }^{n(t)}$ represents the additive white Gauss noise, the mean is zero, variance is $\sigma_{n}^{2}$.

On the basis of this model, we applies PCA on the cyclostationarity features and obtains the principal components of the presence of the primary user or not and obtain characteristic vectors as training samples to form random forest. Then the detecting signal is classified by the trained random forest. As we see from Figure 1.

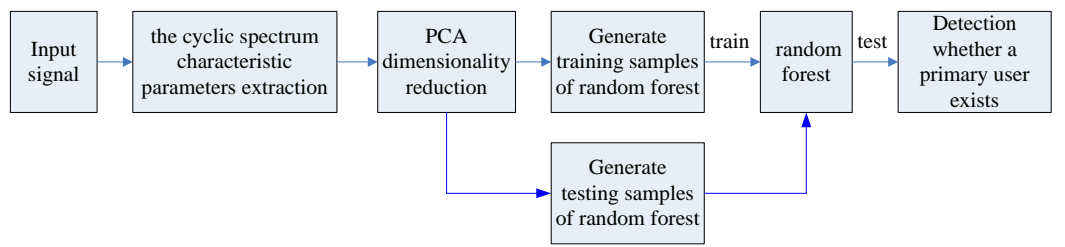

\section{Figure 1. The Model Diagram of Spectrum Sensing Algorithm based on PCA and Random Forest}

\section{Cyclic Spectrum Characteristic Parameters Extraction}

Many signals in communication system exhibit cyclostationarity property called spectral correlation features, these properties can be used for signal classification and detection.

We assume that each secondary user receiving signal is $y(t)$, and it's the autocorrelation function can be expressed as $R_{y}{ }^{\alpha}(\tau)$,

$$
R_{y}^{\alpha}(\tau)=\frac{1}{T_{0}} \int_{0}^{T_{0}} R(t, \tau) e^{-j 2 \pi \alpha t} d t
$$

Where $\alpha$ is cyclic frequency, $\alpha=\frac{1}{T_{0}}, T_{0}$ is cycle period, $R(t, \tau)$ is time-varying cyclic autocorrelation function of $y(t)$,

$$
R(t, \tau)=\lim _{M \rightarrow \infty} \frac{1}{2 M+1} \sum_{m=-M}^{M} y\left(t+m T_{0}+\frac{\tau}{2}\right) y^{*}\left(t+m T_{0}-\frac{\tau}{2}\right)
$$

The cyclic Wiener relation states that the spectral correlation function (SCF) $S_{y}^{\alpha}(f)$ can be obtained from the Fourier transform of $R(t, \tau)$.

$$
S_{y}^{\alpha}(f)=\int_{-\infty}^{\infty} R^{\alpha}(\tau) e^{-j 2 \pi f t} d \tau
$$

We use the spectrally smoothed cyclic periodogram method [15] to calculate $\mathrm{SCF} . S_{y}^{\alpha}(K)$ Is SCF that can be expressed as?

$$
S_{y}{ }^{\alpha}(k)=\frac{1}{T L} \sum_{l=-(L-1) / 2}^{(L-1) / 2} Y\left(k+l+\frac{\alpha}{2}\right) Y^{*}\left(k+l-\frac{\alpha}{2}\right), k=1,2, \cdots, K
$$


Where $Y(k)$ is the discrete Fourier transform of $y(t), L$ is sample length that participate in the frequency domain smoothing, $Y^{*}(k)$ is the conjugate of $Y(k)$.

$$
S(k)=\left.S_{y}^{\alpha}(k)\right|_{\alpha=1 / T_{0}}
$$

For the received signal with more than one circular frequency, it obtains the largest energy spectrum as $S(k)$.

The spectral energy is obtained by

$$
\varepsilon=\frac{1}{K} \sum_{k=0}^{K-1}|S(k)|^{2}
$$

\section{The Algorithm Based on Dimensionality Reduction and Random Forest}

In this section, a novel approach to spectrum sensing is introduced based on the proposed system model.

\subsection{The Procedure of Principal Component Analysis for Dimensionality Reduction}

PCA is the best-known linear dimensionality reduction method. PCA aims to find a subspace $\Omega$ which can maximally retain the variance of the original dataset. The basis of $\Omega$ is obtained by eigen-decomposition of covariance matrix. We assume that the $h_{l 1}, h_{l 2}, \cdots, h_{l M}$ express the $M$ values of cyclic spectrum $S_{y}^{\alpha}(K)$ with $\alpha \neq 0$ respectively. Then we make up a matrix of the original data set $H=\left(h_{l m}\right)_{M \times L}$, where $l$ is the number of samples, $l=1,2, \cdots, L, m=1,2, \cdots, M$. Subsequently, the procedure of PCA for dimensionality reduction can be summarized into the following steps.

Step 1.Standardized the samples of the original data set $H=\left(h_{l m}\right)_{M \times L}$

Assuming the samples of the new data set by standardization can be expressed as $x_{l m}$,

$$
x_{l m}=\frac{h_{l m}-\overline{h_{m}}}{S_{m}}
$$

Where $\overline{h_{m}}=\frac{1}{L} \sum_{l=1}^{L} h_{l m}$ and $S_{m}=\sqrt{\frac{\sum_{l=1}^{L}\left(h_{l m}-\overline{h_{m}}\right)^{2}}{L-1}}$ are the mean and the standard deviation of the original samples respectively.

The new standardization sample matrix

$$
X=\left[X_{1}, X_{2}, \cdots X_{l}\right], X_{l}=\left[x_{l 1}, x_{l 2}, \cdots, x_{l M}\right]^{T} \in R^{M}, l=1,2, \cdots, L \text { is formed. }
$$

Step 2. Compute the covariance matrix of $X$

$$
C=E\left[X X^{T}\right]
$$

The matrix $X X^{T}$ can be expressed by 


$$
\begin{gathered}
X X^{T}=\left[\begin{array}{cccc}
x_{11} & x_{21} & \cdots & x_{L 1} \\
x_{12} & x_{22} & \cdots & x_{L 2} \\
\vdots & \vdots & \ddots & \vdots \\
x_{1 M} & x_{2 M} & \cdots & x_{L M}
\end{array}\right]\left[\begin{array}{cccc}
x_{11} & x_{12} & \cdots & x_{1 M} \\
x_{21} & x_{22} & \cdots & x_{2 M} \\
\vdots & \vdots & \ddots & \vdots \\
x_{L 1} & x_{L 2} & \cdots & x_{L M}
\end{array}\right] \\
=\left[\begin{array}{cccc}
c_{11} & c_{12} & \cdots & c_{1 M} \\
c_{21} & c_{22} & \cdots & c_{2 M} \\
\vdots & \vdots & \ddots & \vdots \\
c_{M 1} & c_{M 2} & \cdots & c_{M M}
\end{array}\right]
\end{gathered}
$$

Where

$$
\begin{aligned}
c_{11}= & x_{11}^{2}+x_{21}^{2}+\cdots+x_{L 1}^{2} \\
c_{12}= & x_{11}^{2}+x_{21} x_{12}+\cdots+x_{L M}^{2} \\
& \cdots \cdots \\
c_{M M}= & x_{1 M}^{2}+x_{2 M}^{2}+\cdots x_{L M}^{2}
\end{aligned}
$$

Thus the covariance matrix of $X$ can be written as

$$
C=\frac{1}{L}\left[\begin{array}{cccc}
c_{11} & c_{12} & \cdots & c_{1 M} \\
c_{21} & c_{22} & \cdots & c_{2 M} \\
\vdots & \vdots & \ddots & \vdots \\
c_{M 1} & c_{M 2} & \cdots & c_{M M}
\end{array}\right]
$$

Step 3. Calculate eigenvalues $\lambda_{1} \geq \lambda_{2} \geq \cdots \geq \lambda_{M}$ and the corresponding eigenvectors $U_{1}, U_{2}, \cdots, U_{M}$ of the covariance matrix $C$.

$$
C U_{l}=\lambda_{m} U_{l} ; \quad m=1,2, \cdots, M
$$

Step 4. The basis of $\Omega$ is $U_{1}, U_{2}, \cdots, U_{K}, K \prec \prec M$. The value of $K$ is determined by the criteria

$$
\frac{\sum_{k=1}^{K} \lambda_{k}}{\sum_{m=1}^{M} \lambda_{m}} \succ \text { threshold }
$$

Usually threshold $\geq 0.85$.

Step 5. Obtain principal components sample matrix $Y_{l}$ by

$$
Y_{l}=X_{l}^{T} U=\left[y_{l 1}, y_{l 2}, \cdots, y_{l K}\right]
$$

Where $U=\left[U_{1}, U_{2}, \cdots, U_{K}\right]$. 
The new samples can be expressed as $Y_{l}^{\prime}=\left[y_{l 1}, y_{l 2}, \cdots, y_{l K}, \varepsilon\right]^{T}$ after dimensionality reduction, $\varepsilon$ expresses the maximum spectral energy of the cyclic spectrum $S_{y}^{\alpha}(K)$ with $\alpha \neq 0$. Then we divide these new samples into training sets and testing sets under the condition of $H_{0}$ and $H_{1}$ respectively.

\subsection{The Procedure of Random Forest for Spectrum Sensing}

According to the spectrum sensing model, we assume that $y_{l 1}^{0}, y_{l 2}^{0}, \cdots, y_{l K}^{0}, \varepsilon^{0}$ are eigenvalues under the condition of $H_{0}$,so characteristic vector expresses $Y_{l}^{0}=\left[y_{l 1}{ }^{0}, y_{l 2}{ }^{0}, \cdots, y_{l K}{ }^{0}, \varepsilon^{0}\right]^{T} ; y_{l 1}^{1}, y_{l 2}^{1}, \cdots, y_{l K}^{1}, \varepsilon^{1}$ are eigenvalues under the condition of $H_{1}$, characteristic vector expresses $Y_{l}{ }^{1}=\left[y_{l 1}{ }^{1}, y_{l 2}{ }^{1}, \cdots, y_{l K}{ }^{1}, \varepsilon^{1}\right]^{T}$. The generating algorithm steps are as follows:

Step 1. In view of the primary user signal, we collect $M_{t}$ of characteristic vectors under the circumstance of presence of primary users as the training positive samples of random forest, and collect $M_{f}$ of characteristic vectors under the circumstance of the absence of primary users as the training negative samples of random forest. We suppose all of $M_{t}$ and $M_{f}$ form a training group $T$.

Step 2. $M_{t}^{c}$ positive samples and $M_{f}^{c}$ negative samples of group $T$ are randomly selected as training samples in order to generated every tree of random forests. Here, we adopt the method of Gini coefficient as the discriminant standard to classify nodes. The Gini index is given by

$$
\operatorname{gini}(T)=1-\sum_{j=1}^{2} P_{j}^{2}
$$

Where $P_{j}$ is probability of the $j-$ th sample? The feature properties that make value of $\operatorname{gini}(T)$ minimum are selected as the nodes' classification properties of tree.

Step 3. The decision trees are not growing until the percentage of the classification purity in each node achieves the desired growth or a given layer. After that, one decision tree in the random forest is generated.

Step 4. Repeat above steps, we can establish random forest with $K$ trees.

Step 5.Then the detecting signal is classified and sensed by the trained random forest.

\section{Simulation Results}

In this section, simulations are used to evaluate the performance of the proposed algorithm with various features. By using BPSK, 2FSK, OFDM signals as input, the resolution of the carrier frequency is $1 \mathrm{MHZ}$, and input signals appear to uniform distribution between 3.1 4.8 GHz.

We compare with MME, SVM, RF, and our proposed algorithm (PCA with RF) under the signal-to-noise ratio $(\mathrm{dB})$ for $-20,-15,-10,-5,0$ circumstances respectively in Matlab7.0. Three cyclic spectrum values and the maximum spectral energy of the cyclic spectrum 
$S_{y}^{\alpha}(K)$ with $\alpha \neq 0$ are chosen to form the training and testing samples of SVM and RF algorithms. The DFT length of cyclic spectrum is 512, and the threshold is 0.95 in the procedure of dimensionality reduction.

In the case of $H_{1}$, we take 600 samples in each signal set of 1000 samples randomly as the training samples to train every tree, and then put them back for the next selection in the training process. Repeat above steps, we can establish random forest with 100 trees. Simulations are carried out $10^{4}$ orders of magnitude times.

The detection rates $(P d)$ of the different algorithms for BPSK varied in low signal-tonoise ratio environments are shown in Figure 2. It is shown that the detection rates performance of the four different algorithms decreases with the decreasing of SNR. The proposed algorithm can achieve 0.96 at $\mathrm{SNR}=-10 \mathrm{~dB}$ (increases $18.0 \%, 33.1 \%$ and $21.8 \%$ compared to MME, SVM and RF algorithms respectively). When the SNR is -20dB, The proposed algorithm can still achieve 0.816, higher than SVM, MME and RF algorithms obviously.

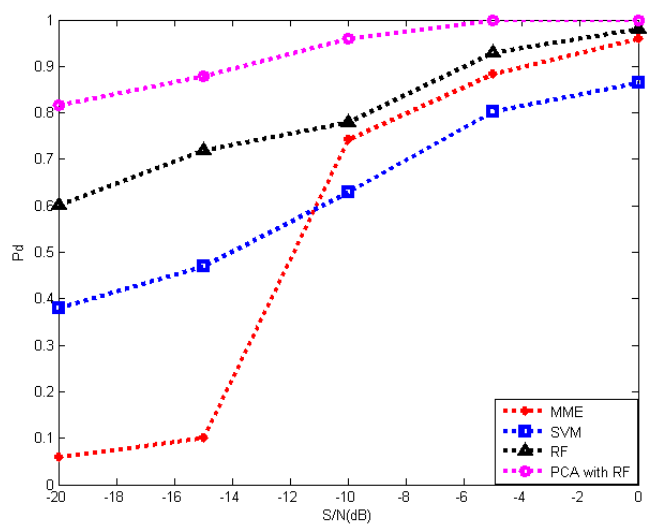

Figure 2. The Detection Rates of the Proposed Algorithm versus MME, RF and SVM for BPSK

Figure 3 shows the detection rates comparison between MME, SVM, RF algorithms and the proposed algorithm for 2FSK.It is observed that the detection rate of proposed algorithm increases from 0.802 to 1 when SNR vary between $-20 \mathrm{~dB}$ to $0 \mathrm{~dB}$ with interval of $5 \mathrm{~dB}$.Under the same varying SNR case, SVM detection rate increases from 0.412 to 0.881 , MME increases from 0.03 to 0.967 , and RF algorithm increases from 0.58 to 0.986 . It shows that the proposed algorithm has high accuracy rate compared to the other algorithms at the low SNR. 


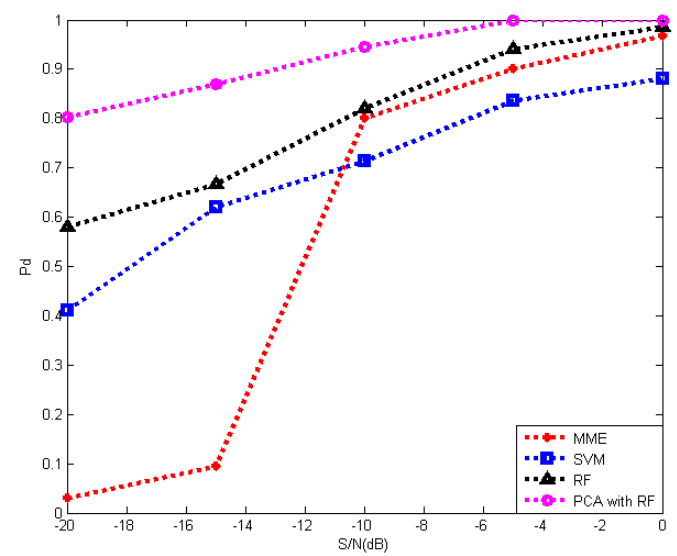

Figure 3. The Detection Rates of the Proposed Algorithm versus MME, RF and SVM for 2FSK

The SVM and RF algorithms with few spectral values and the maximum spectral energy of the cyclic spectrum are not reliable when the SNR is low, because some important information is lost. And MME algorithm reflects the disadvantage of energy detection method that is not suitable in low SNR. Defects of the above algorithms that impact the detection rates performance are overcome effectively in the proposed algorithm. By utilizing the proposed algorithm, most discriminant information and greatly decrease the required feature dimensions in classification can be preserved. The figure also indicates that the random forest algorithm's advantages of the strong classifier synthesized by multiple weak classifiers.

In Figure 4, we repeat the same comparison between the above algorithms for OFDM. Under the low SNR environment, our algorithm still outperforms the other three algorithms.

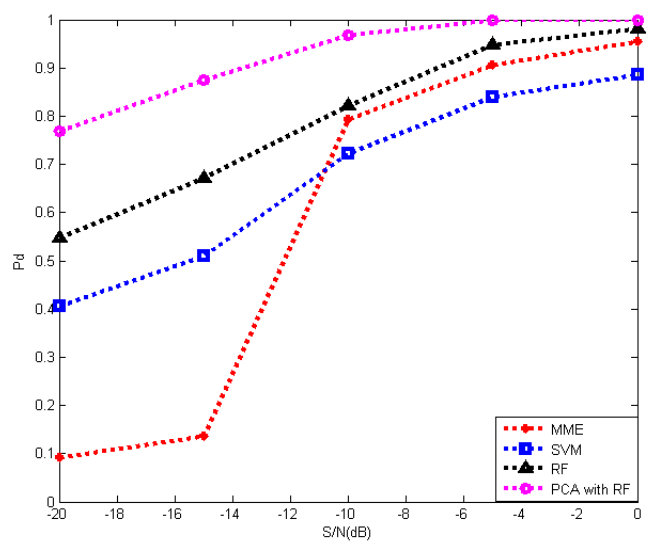

Figure 4. The Detection Rates of the Proposed Algorithm versus MME, RF and SVM for OFDM

The average error rates $\left(P_{w}\right)$ of our proposed algorithm compare with MME, SVM, RF for different modulated signals are shown in Figure 5. The average error rates performance of the four different algorithms decreases with the increasing of SNR. The average error rates of the proposed algorithm decreases from 0.205 to 0 when SNR vary between $-20 \mathrm{~dB}$ to $0 \mathrm{~dB}$ 
with interval of 5dB lower than SVM and MME algorithms under the same varying SNR case obviously. The results show that the proposed algorithm has low average error rate compared to the other algorithms in the low SNR.

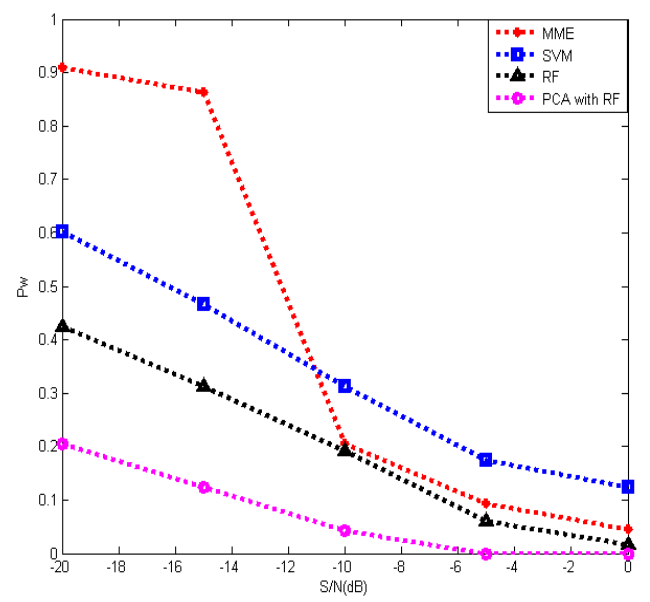

Figure 5. The Average Error Rates of our Proposed Algorithm versus MME，RF and SVM

In order to show the variation between the detection rates performance of the proposed algorithm with the number of dimension, the experiments are carry through dimensionality $N=1,2,3, \cdots, 13$ in $\mathrm{SNR}=-10 \mathrm{~dB}$, which are obtained by the different threshold in PCA. The results are shown in Figure 6, it is verify the fact that more and more dimensions of the data are included (more information are included), the detection rates should become higher and higher globally. On the other hand, by dimensionality reduction, most of the useful information can be extracted to the first dimension. Therefore, even if we increase the dimensions of the reduced data, the detection rates do not embody obvious improvement. The same results are also reflected in the experiments in the different SNR environments.

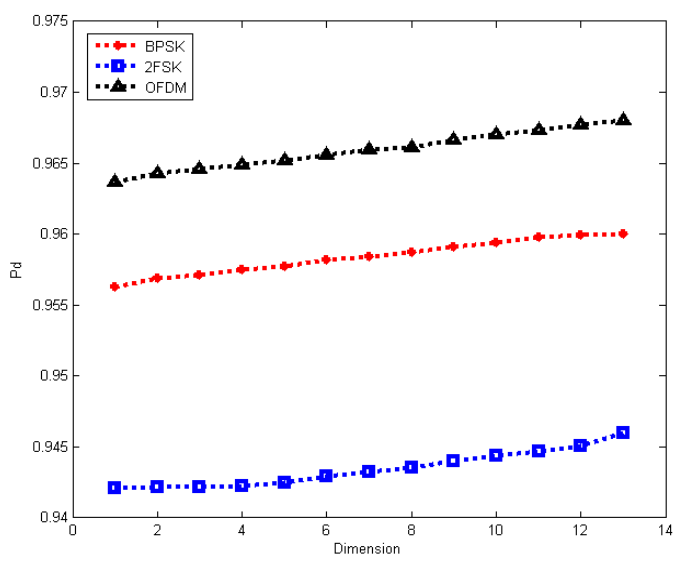

Figure 6. The Detection Rates of the Proposed Algorithm with the Number of Dimension in SNR=-10dB 


\section{Conclusion}

In this paper, we propose a novel approach combining PCA and random forest to detect the primary user signal in low SNR. A set of cyclic spectrum features of the received radio signal are first calculated, and a principal component analysis (PCA) is applied to extract the most discriminant feature vector for classification. Then random forest (RF) is generated to classify the detecting signal. The experimental results show that the proposed algorithm solves the problem of the low accuracy on detection of the weak primary users in the low SNR environment effectively. Defects of inadequate feature extraction that impact the primary user spectrum sensing are overcome effectively in the proposed algorithm, which demonstrates the feasibility of our algorithm.

\section{Acknowledgments}

This work is partly supported by the National Natural Science Foundation of China under Grant (No.61374097), (No.61004052), Hebei Province Natural science fund under Grant (No. F2011501021) and the Science and Technology Research Project of Higher Education of Hebei Province under Grant (QN20132010) respectively.

\section{References}

[1] B. Wang and K. J. R. Liu, "Advances in cognitive radio networks: A survey", IEEE Journal of Selected Topics in Signal Processing, vol. 5, no. 1, (2011).

[2] W. Xin, W. Jinkuan and L. Zhigang, "Spectrum sensing algorithm based on random forest in cognitive network", Chinese Journal of Scientific Instrument, vol. 34, no. 11, (2013).

[3] Y. C. Liang, K. C. Chen and G. Y. Li, "Cognitive Radio Networking and Communications: An Overview", IEEE Transactions on Vehicular Technology, vol. 60, no. 7, (2011).

[4] I. F. Akyildiz, W. Y. Lee and M. C. Vuran, "Next generation/dynamic spectrum access/ cognitive radio wireless networks: a survey", Computer Networks, vol. 50, no. 13, (2006).

[5] Y. Zeng and Y. Liang. "Maximum-minimum eigenvalue detection for cognitive radio", Proc of IEEE 18th International Symposium on Personal, Indoor and Mobile Radio Communications(PIMRC 2007), Athens, Greece, (2007) September, pp. 1-5.

[6] P. D. Sutton, K. E. Nolan and L. E. Doyle, "Cyclostationary signatures in practical cognitive radio applications", IEEE Journal of Selected Areas in Communications, vol. 26, no. 1, (2008).

[7] H. Sun, A. Nallanathan and C. X. Wang, "Wideband spectrum sensing for cognitive radio networks: a survey ", IEEE Journal of Wireless Communications, vol. 20, no. 2, (2013).

[8] M. Bkassiny, Y. Li and S. Jayaweera, "A Survey on Machine-Learning Techniques in Cognitive Radios", IEEE Journal of Communications Surveys \& Tutorials, vol. 45, no. 5, (2012).

[9] N. Baldo, B. Tamma, B. Manojt, R. Rao and M. Zorzi, "A neural network based cognitive controller for dynamic channel selection”, Proc of IEEE International Conference on Communications (ICC '09), Dresden, Germany, (2009) June, pp. 1-5.

[10] V. Tumuluru, P. Wang and D. Niyato, "A neural network based spectrum prediction scheme for cognitive radio", Proc of IEEE International Conference on Communications (ICC'10), Cape Town, South Africa, (2010) May, pp. 1-5.

[11] V. N. Vapnik, "Statistical Learning Theory", Wiley, New York, (1998).

[12] H. Hu, J. Song and Y. Wang, "Signal classification based on spectral correlation analysis and SVM in cognitive radio", Proc of the 22st International Conference on Advanced Information Networking and Applications (AINA '08), Mar, Okinawa, Japan, (2008), pp. 883-887.

[13] L. Breiman, "Random forests", Machine Learning, vol. 45, (2001).

[14] S. Wold, K. Esbensen and P. Geladi, "Principal component analysis", Chemometrics and Intelligent Laboratory Systems, vol. 2, (1987).

[15] R. S. Roberts, W. A. Brown and Jr. H. H. Loomis, "Computationally Efficient Algorithms for Cyclic Spectral Analysis”, IEEE Journal of Signal Processing Magazine, vol. 8, no. 2, (1991). 